\title{
Regulatory effects of miRNA on gastric cancer cells
}

\author{
OUYANG YANG, JIANHUA HUANG and SUN LIN \\ Department of General Surgery, Xiangya Hospital of Central-South University, Changsha, Hunan 410008, P.R. China
}

Received August 19, 2013; Accepted March 4, 2014

DOI: $10.3892 / \mathrm{ol} .2014 .2232$

\begin{abstract}
The present study aimed to screen the regulatory mechanism of microRNA (miR/miRNA) typing on gastric cancer cells in gastric cancer tissues. In total, 46 patients who underwent gastric resection in Xiangya Hospital of Central-South University (Changsha, Hunan, China) between January and December 2012 were selected. Gastric cancer cells were obtained for RNA extraction, and miRNAs were detected by quantitative polymerase chain reaction. Compared with the expression levels in the normal gastric mucosa, the expression levels of miR-9, miR-433, miR-19b and miR-370 were downregulated; the differences was statistically significant, except for miR-19b $(\mathrm{P}<0.05)$. miRNAs play a regulative role in the occurrence and development of gastric cancer, and changes in their expression provide a basis for the diagnosis of this disease.
\end{abstract}

\section{Introduction}

Gastric cancer is one of the most common malignant tumors in the digestive tract. In China, almost 300,000 individuals succumb to gastric cancer and 400,000 new cases are diagnosed each year (1). The diagnosis, metastasis and prognosis of tumors use various molecular markers, so the search for molecular markers at different stages of tumor development is of great clinical significance to the early diagnosis, recurrence, metastasis prediction and prognosis of tumors (2). Studies on tumor markers have been carried out for numerous years, and there are also a small number of markers used in the clinic, but their sensitivity and specificity remain extremely limited $(3,4)$. Therefore, it is essential to study the molecular markers associated with gastric cancer metastasis and prognosis. The emergence of microRNA (miR/miRNA) provides a novel concept for cancer research and the screening of molecular markers.

miRNAs are a class of endogenous non-coding small molecule RNAs with regulatory function on genes, which are 22-28 nucleotides in length and widely exist in eukaryotes (5). A gene with miRNA encoded in the nucleus is first transcribed

Correspondence to: Dr Sun Lin, Department of General Surgery, Xiangya Hospital of Central-South University, 84 Xiangya Lu Road, Changsha, Hunan 410008, P.R. China

E-mail: sunlindgs@163.com

Key words: gastric cancer cell, gene regulation, microRNA into a pri-miRNA, i.e., the precursor of miRNA (6). Specific miRNAs have a close association with tumors. It has been confirmed that there are 500 miRNAs in the human genome, of which at least 200 miRNA sequences are closely associated with tumors, and a number of them can be used as tumor molecular markers (7). miRNAs as molecular markers have unique advantages; they are more stable compared with mRNA, are not affected by other physiological states of the body, can be amplified and can be more easily detected compared with proteins (8).

The present study aimed to conduct screening, identification and validation of miRNAs in gastric cancer tissues and cell lines, combined with an analysis on the clinicopathological factors and survival data of gastric cancer patients. The study also aimed to reveal the regulation mechanism of miRNA expression in gastric cancer, in order to provide a theoretical basis for miRNAs to become novel molecular markers and therapeutic targets of the metastasis and prognosis of gastric cancer.

\section{Materials and methods}

Materials. The following materials were used in the present study: A normal gastric mucosal epithelial cell line, GES-1 (Shanghai Cell Bank Center, Chinese Academy of Medical Sciences, Shanghai, China), a gel imager and electrophoresis apparatus (Bio-Rad, Hercules, CA, USA) and pipettes (5, 10 and $200 \mu \mathrm{l}$ and $1 \mathrm{ml}$; Eppendorf, Hamburg, Germany).

Subjects. A total of 46 patients who underwent gastric resection in Xiangya Hospital of Central-South University (Changsha, Hunan, China) between January and December 2012 were selected. In total, thee study group consisted of 26 males and 20 females aged between 30 and 70 years old, with the mean age of $52.1 \pm 6.7$ years old. The clinical data of the patients with gastric cancer were recorded completely, including pathological type, stage, metastasis, one-, two- and three-year survival rates and other detailed information. The study was approved by the ethics comittee of Xiangya Hospital of Central-South University. Consent was obtained prior to examination for the enrolled patients according to the Declaration of Helsinki and relevant laws in China. All treatments were performed based on the best interests of the patients.

RNA extraction. The gastric cancer cells were plated in 6 -well culture plates $24 \mathrm{~h}$ prior to transfection. When the cells reached $30-50 \%$ confluency, $10 \mu \mathrm{l}$ antisense oligonucleotide liposome (Promega Corporation, Madison, WI, USA) was 


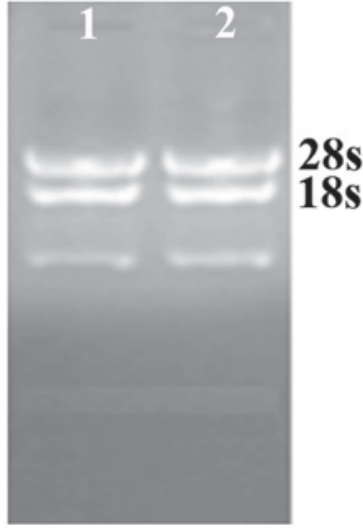

Figure 1. RNA extraction results.

taken correspondingly and diluted to $250 \mu \mathrm{l}$ by Opti-minimal essential medium (Invitrogen China Limited, Beijing, China) for action at room temperature for $10 \mathrm{~min}$. The two were then slowly mixed prior to leaving the mixture stationary for $30 \mathrm{~min}$ and finally added it to the cells, which were agitated and mixed well. Following $12 \mathrm{~h}$ of culture, the medium was replaced with RPMI-1640 containing 10\% fetal bovine serum for $48 \mathrm{~h}$. Subsequently, the medium was aspirated, the cells were washed with phosphate-buffered saline and TRIzol (Invitrogen Life Technologies, Carlsbad, CA, USA) was added. The samples were then stored at $-70^{\circ} \mathrm{C}(9,10)$.

Quantitative polymerase chain reaction ( $q P C R)$. The detection of the miRNA target and internal control, U6, was conducted in a Rotor-Gene 3000 Real-time PCR machine (Corbett Research, Mortlake, Australia). The $25 \mu 1$ reaction system included 2.5 $\mu \mathrm{l}$ dNTP, $2.5 \mu \mathrm{l}$ 10X PCR buffer, $1.5 \mu 1 \mathrm{MgCl}_{2}$ aqueous solution, 1 unit Taq enzyme, SYBR-Green I with a final concentration of $0.25 \mathrm{X}, 1 \mu \mathrm{l}$ PCR specific primer, $1 \mu 1$ reverse transcriptase product and RNA-free enzyme water. The reaction conditions were as follows: $95^{\circ} \mathrm{C}$ for $5 \mathrm{~min}$, followed by $95^{\circ} \mathrm{C}$ for $10 \mathrm{sec}$ and $60^{\circ} \mathrm{C}$ for $1 \mathrm{~min}$ for a total of 40 cycles. The miRNA expression level was detected with the threshold cycle number $(\mathrm{Ct})$. The miRNA targets were relatively quantified by the $\Delta \Delta \mathrm{Ct}$ method. $\Delta \mathrm{Ct}$ refers to the $\mathrm{Ct}$ value of the miRNA target minus the $\mathrm{Ct}$ value of U6RNA, and $\Delta \Delta \mathrm{Ct}$ is the value of the $\Delta \mathrm{Ct}$ value of each gastric tissue sample minus the $\Delta \mathrm{Ct}$ value of the normal gastric tissue sample. The changing multiple was calculated using the $2^{-\Delta \Delta C t}$ formula (11).

Statistical analysis. All data were analyzed by SPSS, version 13.0 (SPSS, Inc., Chicago, IL, USA). The measurement data were expressed as the mean \pm standard deviation and processed by paired t-test and compatibility group design analysis of variance. $\mathrm{P}<0.05$ was considered to indicate a statistically significant difference.

\section{Results}

RNA extraction results. The integrity of RNA can be assessed by electrophoresis on a denaturing agarose gel. Sharp $28 \mathrm{~S}$ and 18S RNA bands (eukaryotic samples) are produced by intact total RNA that is run on a denaturing gel. An example for RNA electrophoresis on a denaturing agarose gel is shown in Fig 1.

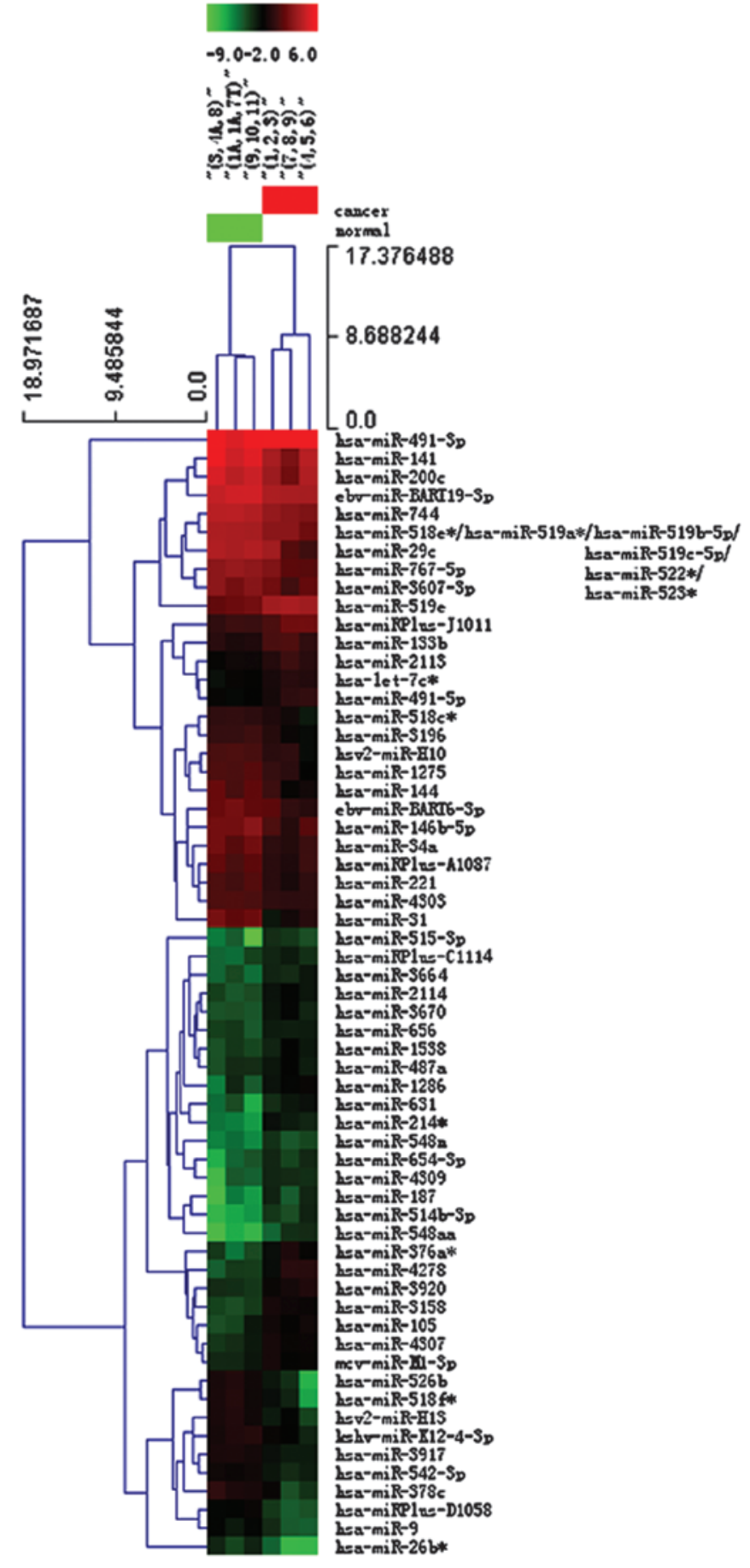

Figure 2. Hierarchical clustering for differentially-expressed miRNAs in a cancer vs. normal pass volcano plot (fold-change $\geq 2.0$ ). Red indicates high relative expression and green indicates low relative expression. $\mathrm{miRNA} / \mathrm{miR}$, microRNA.

The RNA electrophoresis showed that the 28S and 18S bands were wider compared with the control (Fig. 1), indicating that the RNA was not degraded.

Heat map and hierarchical clustering. Two-way hierarchical clustering results for the miRNA and samples are shown in Fig. 2 by the heat map diagram. One miRNA is represented per row, and one sample per column. The left-hand side of the image shows the miRNA clustering tree, while the sample clustering tree appears at the top. Samples and miRNAs 
Table I. Expression levels of miRNAs in gastric cancer and normal gastric mucosa tissues.

\begin{tabular}{lcccr}
\hline Tissue & miR-9 & miR-19b & $\operatorname{miR}-433$ & $\mathrm{miR}-370$ \\
\hline Cancer & $0.419 \pm 0.211^{\mathrm{a}}$ & $0.648 \pm 0.351^{\mathrm{a}}$ & $0.397 \pm 0.284^{\mathrm{a}}$ & $0.504 \pm 0.351^{\mathrm{a}}$ \\
Normal & $0.985 \pm 0.429$ & $1.051 \pm 0.589$ & $1.061 \pm 0.514$ & $0.991 \pm 0.512$ \\
\hline
\end{tabular}

${ }^{\mathrm{a}} \mathrm{P}<0.05$ vs. normal. miR/miRNA, microRNA.

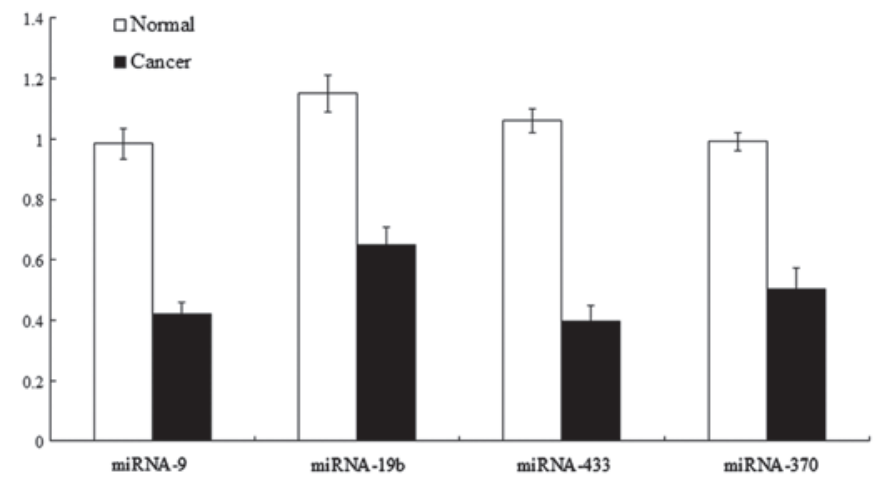

Figure 3. Expression levels of miRNAs in gastric cancer and normal gastric mucosa tissues. miRNA, microRNA.

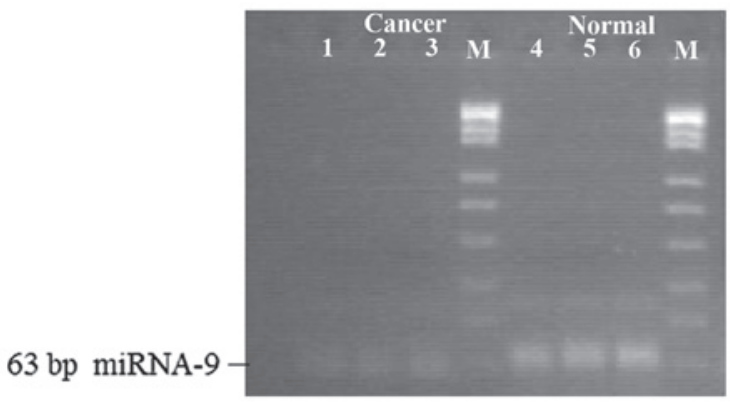

Figure 4. Expression levels of miR-9 in gastric cancer and normal gastric mucosa tissues. miR, microRNA.

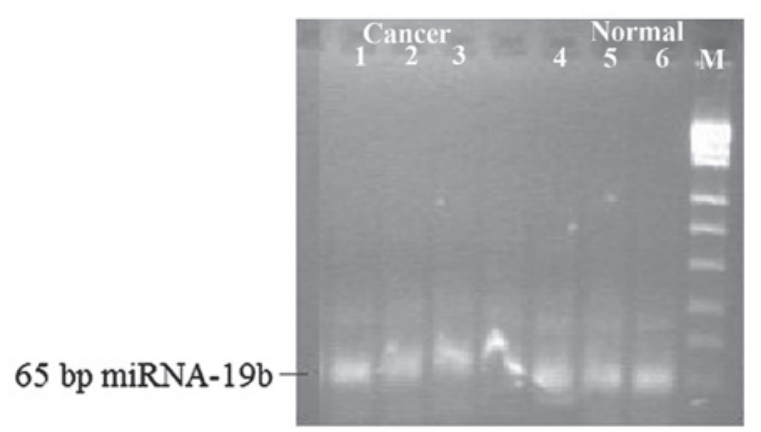

Figure 5. Expression levels of miR-19b in gastric cancer and normal gastric mucosa tissues. miR, microRNA.

are arranged by cluster analysis into groups based on their expression levels, which enables hypotheses to be generated regarding the correlations between the miRNAs and samples. Subsequently, hierarchical clustering was performed based on the differentially-expressed miRNAs in a cancer vs. normal

\section{4 bp miRNA-433}

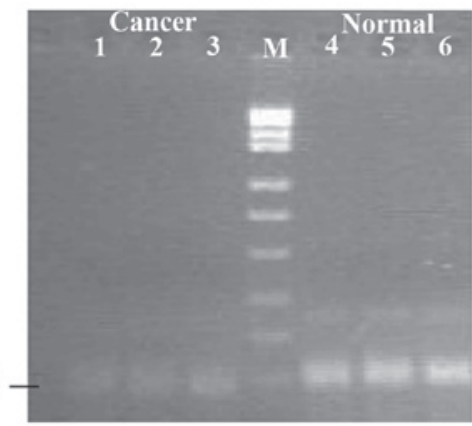

Figure 6. Expression levels of miR-433 in gastric cancer and normal gastric mucosa tissues. miR, microRNA.

\section{3 bp miRNA-370}

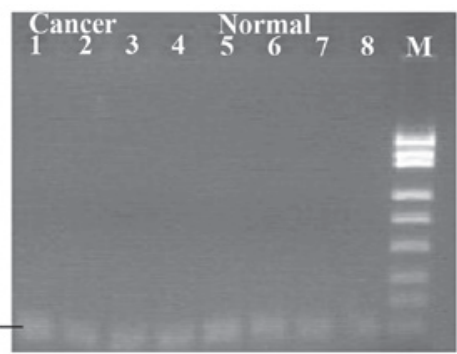

Figure 7. Expression levels of miR-370 in gastric cancer and normal gastric mucosa tissues. miR, microRNA.

pass volcano plot. The result of hierarchical clustering shows distinguishable miRNA expression profiling among the samples (Fig. 2).

miRNA expression in gastric cancer tissues. The qPCR result showed that compared with the normal gastric mucosa, the expression levels of miR-9, miR-433, miR-19b and miR-370 were detected in the gastric cancer tissues and cells (Table I and Fig. 3), and the electrophoretic bands were located at $63,64,65$ and $63 \mathrm{bp}$. Compared with levels in the normal gastric mucosa, the miR-9, miR-433, miR-19b and miR-370 expression levels were lowered (Figs. 4-7).

Association between miRNA expression in gastric cancer and clinical pathological factors. Downregulated miR-9 expression was associated with the size of the foci and lymph node metastasis $(\mathrm{P}=0.036$ and 0.028 , respectively), while decreased miR-433 expression was associated with the foci site and pathological grading ( $\mathrm{P}=0.003$ and 0.005 , respectively). Reduced miR-19b expression was correlated with the foci site and pathological grading $(\mathrm{P}=0.002$ and 0.009 , respectively), and downregulated miR-370 expression was associated with 


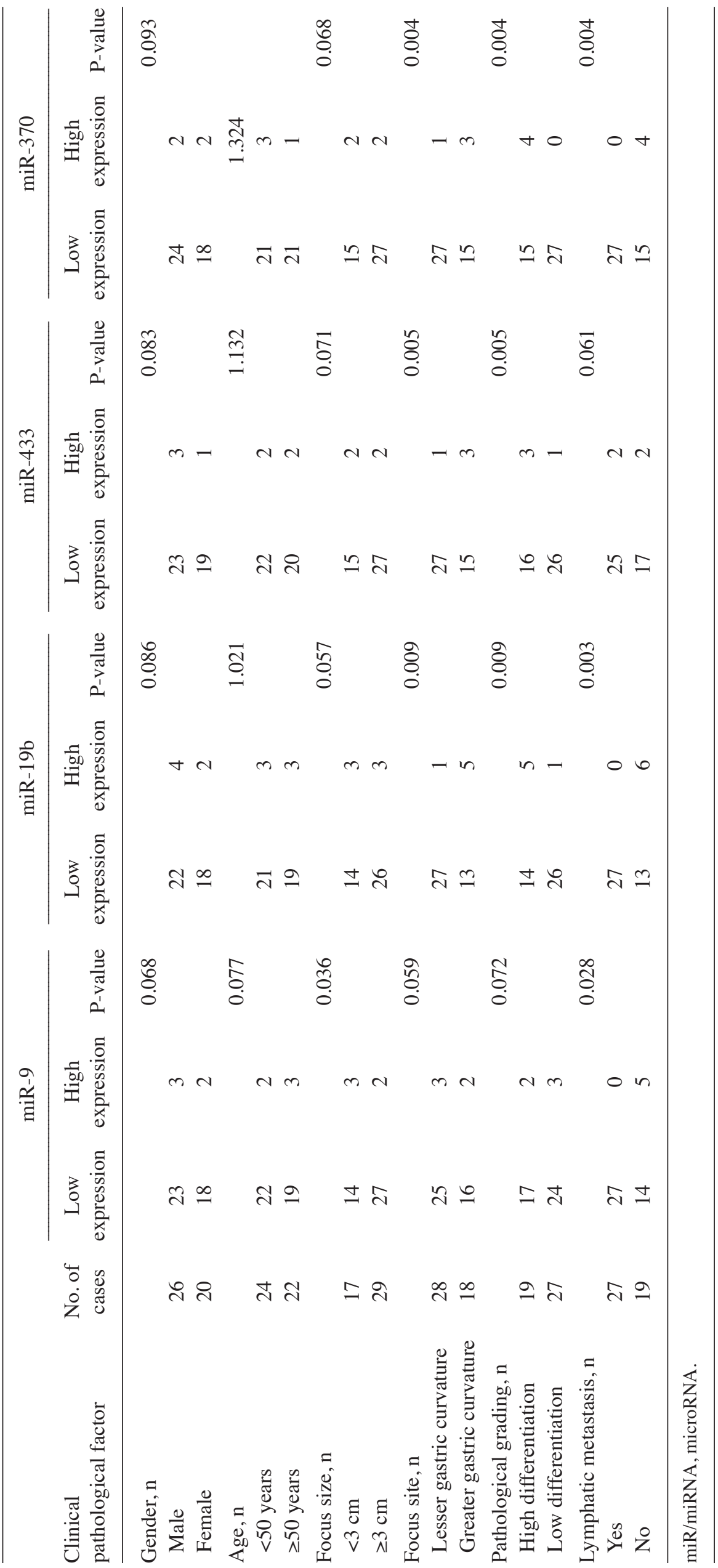


the foci site, pathological grading and lymph node metastasis $(\mathrm{P}=0.003,0.004$ and 0.004 , respectively) (Table II).

\section{Discussion}

There are various molecular markers that can be used for tumor diagnosis, metastasis and prognosis, therefore, the search for tumor molecular markers at these differing stages provides an important link between basic and clinical research, and a main way for basic research to serve a function in the clinic (12-14). Studies have shown that the metastasis and prognosis of gastric cancer is closely associated with the connexins (Cx). The mRNA and protein levels of Cx32 and $\mathrm{Cx} 43$ in gastric cancer cells and tissues are significantly reduced, and thus miRNAs that regulate $\mathrm{Cx}$ are molecular markers of gastric cancer $(15,16)$. Through the process of looking for miRNA-9, $-433,-19 \mathrm{~b}$ and -370 which regulate $\mathrm{Cx}$ and via the use of statistical analysis on metastasis and prognosis in clinical groups, combined with clinical data, the present study confirmed that the miRNAs are good molecular markers. The present study is of great significance in the theoretical basis of the development, metastasis and prognosis of gastric cancer.

pri-miRNA is sheared into a length of $\sim 70$ nucleotides under the effect of the double-stranded RNA specific nuclease-Drosha RNase, then pri-miRNA, having a hairpin structure, is transferred from the nucleus into the cytoplasm with the action of the transporter protein, exportin 5, and cut into double-stranded miRNAs that are 21-25 nucleotides in length under the effect of another double-stranded RNA-specific nuclease, the Dicer enzyme (17-19). Subsequent to the unwinding of the double helix, one of the two chains connects with the RNA-induced silencing complex (RISC), functioning as an miRNA, and the other chain is degraded immediately. The combination of miRNAs with target mRNA can mediate the degradation of RISC into target fragments or hinder the translation process, which depends on the degree of mismatch between miRNA and the target mRNA $3^{\prime}$ untranslated region. If it is a complete or almost complete match, the RNA interference pathway can be induced and mRNA will be degraded. As the majority of miRNAs are not completely complementary with target mRNAs, which only play a role in closing mRNA targets and inhibiting the translation process so as to regulate the expression of target genes, miRNA plays a vital role in a variety of biological processes, including cell proliferation, differentiation and apoptosis (20-22).

In addition, through the application of gene-chip technology and bio-informatics methods, certain miRNAs that are closely associated with gastric cancer have been found. The experimental study by Li et al (23) showed that in $92 \%$ of gastric cancer samples, miR-21 had a significantly high expression level, indicating that miR-21 may serve as an effective diagnostic marker of gastric cancer. He and Wang (24) identified that in gastric cancer cell lines, the expression of the tumor-associated protein, high-mobility group AT-hook 2 (HMGA2), was inhibited by miRNA let27. In gastric cancer cells with a low HMGA2 expression level, the expression level of miRNA let27a, let27b and let27c was significantly higher compared with gastric cancer cells with a high HMGA2 expression level, indicating that miRNAs may play a biological role to promote or inhibit tumor development through the interaction between tumor-associated proteins.

The main factor affecting the prognosis of gastric cancer is the invasion and metastasis of gastric cancer, therefore, it is of great significance to study particular miRNAs that are closely associated with gastric cancer invasion, metastasis and development, which can also provide clues for the screening of miRNA molecular markers. A study by Li et al (25) analyzed the effect of miR-10a on the migration and invasion of the gastric cancer BGC823 cell line, and found that the transfection of miR-10a had no significant effect on the proliferation and apoptosis of BGC 823 , but could clearly promote its migration and invasion. Transfection with mature human miR-210a could increase the miR-10a expression in BGC823 and significantly promote the migration and invasion of BGC823 $(26,27)$. Chen et al $(28)$ studied the association between miRNA and RPL23 (a lymph node metastasis-related gene) by creating an miRNA expression plasmid that could express miR-155. This effectively silenced the expression of the PRL23 target gene in the gastric cancer SGC7901 cell line and significantly inhibited the invasion and metastasis of the SGC7901 cells. Therefore, miRNAs are closely associated with tumor proliferation and apoptosis, and their correlation with tumor invasion and metastasis requires further research.

To understand the carcinogenic mechanism of miRNAs, systematic identification of the target taking effect in vivo is required. The bioinformatics data show that each miRNA can regulate hundreds of target genes. It is estimated that $30 \%$ of human genes are subject to regulation by miRNAs, which also indicates that miRNA may affect all the signaling pathways (29). A study by Cao et al (30) selected myocardial cells as the study object and found that the regulatory targets of miR-1 existed in the GJA1-encoded Cx43, and miR-1 was overexpressed in myocardial cells and inhibited the expression of GJA1. Cx43 expression is reduced accordingly in the myocardium, aggravating arrhythmias (31). In this study, the expression of miRNA in gastric cancer tissues and cells was evaluated by analyzing the clinical pathological factors and survival data of patients. The findings revealed the mechanism regarding the mechanism of action, and provide theoretical evidence for the use of miRNA as novel molecular markers and treatment targets for the metastasis and prognosis of gastric cancer.

\section{References}

1. Tavazoie SF, Alarcón C, Oskarsson T, et al: Endogenous human microRNAs that suppress breast cancer metastasis. Nature 451: 147-152, 2008.

2. Waldman SA and Terzic A: MicroRNA signature as diagnostic and therapeutic targets. Clin Chem 54: 943-944, 2008.

3. Ito T, Shimada Y, Kan T, et al: Pituitary tumor-transforming 1 increases cell motility and promotes lymph node metastasis in esophageal squamous cell carcinoma. Cancer Res 68: 3214-3224, 2008.

4. Inose $\mathrm{H}$, Ochi $\mathrm{H}$, Kimura A, et al: A microRNA regulatory mechanism of osteoblast differentiation. Proc Natl Acad Sci USA 106: 20794-20799, 2009.

5. Harquail J, Benzina S and Robichaud GA: MicroRNAs and breast cancer malignancy: an overview of miRNA-regulated cancer processes leading to metastasis. Cancer Biomark 11: 269-280, 2012.

6. Huang XA and Lin H: The miRNA regulation of stem cells. Wiley Interdiscip Rev Membr Transp Signal 1: 83-95, 2012. 
7. Maccani MA and Marsit CJ: Exposure and fetal growth-associated miRNA alterations in the human placenta. Clin Epigenetics 2: 401-404, 2011.

8. Jethwa K, Wei J, McEnery $\mathrm{K}$ and Heimberger $\mathrm{AB}$ : miRNA-mediated immune regulation and immunotherapeutic potential in glioblastoma. Clin Investig (Lond) 1: 1637-1650, 2011.

9. Lodewijk L, Prins AM, Kist JW, Valk GD, Kranenburg O, Rinkes IH and Vriens MR: The value of miRNA in diagnosing thyroid cancer: a systematic review. Cancer Biomark 11: 229-238, 2012.

10. Sanchez N, Lao N, Gallagher C, Clynes M and Barron N: Engineering $\mathrm{CHO}$ cell growth by stable manipulation of miRNA expression. BMC Proc 5 (Suppl 8): P22, 2011.

11. Wang L, Liu W, Jiang W, Lin J, Jiang Y, Li B and Pang D: A miRNA binding site single-nucleotide polymorphism in the 3'-UTR region of the IL23R gene is associated with breast cancer. PLoS One 7: e49823, 2012.

12. Hirata H, Ueno K, Shahryari V, Tanaka Y, Tabatabai ZL, Hinoda Y and Dahiya R: Oncogenic miRNA-182-5p targets Smad4 and RECK in human bladder cancer. PLoS One 7: e51056, 2012.

13. Srivastava K and Srivastava A: Comprehensive review of genetic association studies and meta-analyses on miRNA polymorphisms and cancer risk. PLoS One 7: e50966, 2012.

14. Agirre X, Martínez-Climent JÁ, Odero MD and Prósper F: Epigenetic regulation of miRNA genes in acute leukemia. Leukemia 26: 395-403, 2012.

15. Yue SB, Trujillo RD, Tang Y, O'Gorman WE and Chen CZ: Loop nucleotides control primary and mature miRNA function in target recognition and repression. RNA Biol 8: 1115-1123, 2011.

16. Guo Y, Chen Y, Carreon S and Qiang M: Chronic intermittent ethanol exposure and its removal induce a different miRNA expression pattern in primary cortical neuronal cultures. Alcoho Clin Exp Res 36: 1058-1066, 2012.

17. Martin EC, Bratton MR, Zhu Y, Rhodes LV, Tilghman SL, Collins-Burow BM and Burow ME: Insulin-like growth factor-1 signaling regulates miRNA expression in MCF-7 breast cancer cell line. PLoS One 7: e49067, 2012.

18. Devaraj S and Natarajan J: miRNA-mRNA network detects hub mRNAs and cancer specific miRNAs in lung cancer. In Silico Biol 11: 281-295, 2012 .

19. Alshalalfa M: MicroRNA response elements-mediated miRNA-miRNA interactions in prostate cancer. Adv Bioinformatics 2012: 839837, 2012.
20. Arão TC, Guimarães AL, de Paula AM, Gomes CC and Gomez RS: Increased miRNA-146a and miRNA-155 expressions in oral lichen planus. Arch Dermatol Res 304: 371-375, 2012.

21. Staton AA and Giraldez AJ: Use of target protector morpholinos to analyze the physiological roles of specific miRNA-mRNA pairs in vivo. Nat Protoc 6: 2035-2049, 2011.

22. Fabbri E, Brognara E, Borgatti M, et al: miRNA therapeutics: delivery and biological activity of peptide nucleic acids targeting miRNAs. Epigenomics 3: 733-745, 2011

23. Li SC, Liao YL, Ho MR, Tsai KW, Lai CH and Lin WC: miRNA arm selection and isomiR distribution in gastric cancer. BMC Genomics 13 (Suppl 1): S13, 2012.

24. He M and Wang ZW: Current status and development of miRNA and siRNA research on gastric cancer. Yi Chuan 33: 925-930, 2011 (In Chinese).

25. Li C, Li JF, Cai Q, Qiu QQ, Yan M, Liu BY and Zhu ZG: miRNA-199a-3p in plasma as a potential diagnostic biomarker for gastric cancer. Ann Surg Oncol 20 (Suppl 3): S397-S405, 2013.

26. Budak H and Akpinar A: Dehydration stress-responsive miRNA in Brachypodium distachyon: evident by genome-wide screening of microRNAs expression. OMICS 15: 791-799, 2011.

27. Rao E, Jiang C, Ji M, et al: The miRNA-17 92 cluster mediates chemoresistance and enhances tumor growth in mantle cell lymphoma via PI3K/AKT pathway activation. Leukemia 26: 1064-1072, 2012

28. Chen W, Tang Z, Sun Y, et al: miRNA expression profile in primary gastric cancers and paired lymph node metastases indicates that miR-10a plays a role in metastasis from primary gastric cancer to lymph nodes. Exp Ther Med 3: 351-356, 2012.

29. Tonevitsky AG, Maltseva DV, Abbasi A, Samatov TR, Sakharov DA, Shkurnikov MU, et al: Dynamically regulated miRNA-mRNA networks revealed by exercise. BMC Physiol 13, 9, 2013.

30. Cao W, Fan R, Wang L, et al: Expression and regulatory function of miRNA-34a in targeting survivin in gastric cancer cells. Tumour Biol 34: 963-971, 2013.

31. Schnitger AK, Machova A, Mueller RU, et al: Listeria monocytogenes infection in macrophages induces vacuolar-dependent host miRNA response. PLoS One 6: e27435, 2011. 